This is a peer-reviewed, accepted author manuscript of the following article: Theodorou, I., Clemente, C., \& Vasile, M. (2017). Space object detection and characterisation with a passive space-borne bistatic radar. In 68th International Astronautical Congress, IAC 2017: Unlocking Imagination, Fostering Innovation and Strengthening Security (pp. 341-345). (Proceedings of the International Astronautical Congress, IAC; Vol. 1). International Astronautical Federation (IAF).

\title{
Space Object Detection and Characterisation with a Passive Space-borne Bistatic Radar Ilias Theodorou ${ }^{\text {a* }}$, Carmine Clemente ${ }^{\mathrm{a}}$, Massimiliano Vasile ${ }^{\mathrm{b}}$
}

\author{
${ }^{a}$ Department of Electronic and Electrical Engineering, University of Strathclyde, 204 George St, Glasgow G1 1XW, \\ UK, ilias.theodorou@strath.ac.uk, carmine.clemente@strath.ac.uk . \\ ${ }^{\mathrm{b}}$ Department of Mechanical and Aerospace Engineering, University of Strathclyde, 75 Montrose Street, Glasgow, \\ G11X,UK, massimiliano.vasile@strath.ac.uk \\ * Corresponding Author
}

\begin{abstract}
This paper presents a feasibility study and performance assessment of a simple and low-cost system to detect, characterise and potentially track space debris in Low Earth Orbit. The concept exploits any Earth-orbiting satellite broadcasting radio waves as illuminators of a bi-static radar system in which the receiver is equipped of a small antenna on board of a very low altitude orbiting CubeSAT. In fact, in principle the receiver can be installed on any low-altitude satellite (or even the International Space Station) and does not require a dedicated mission. Alternatively, it can be demonstrated that one can install the receiver on one or more CubeSATs flying at very low altitude (below 400km) and provide a dedicated service that does not increase the debris population. The paper will investigate the use of different illuminators at different frequencies. It will be shown that, depending on the integration time that ultimately depends on the time illuminator receiver and target are in view, one can detect objects down to few centimetres in size and recognize targets observed previously.
\end{abstract}

Keywords: (space debris, cubeSAT, passive bistatic radar).

\section{Introduction}

Space activities through all these years have resulted to a dangerous problem known as space debris. More than half a million leftover pieces of junk orbiting the Earth are tracked, travelling at speeds fast enough to occur catastrophic damages to a satellite or a spacecraft [1]. These numbers are continuously gowning, increasing the risk of existing or future missions. An example of this risk is the Iridium-Cosmos collision in 2009, which was a nodal point of this situation [2].

Nowadays, a combination of ground and space-based measurements provide information about space debris. The United States Space Command (USSPACECOM) [3] using a radar network of radars and telescopes tracks unknown orbital objects whereas, European Space Agency (ESA) with a new program called European Space Situational Awareness System (ESSAS) in 2009, can track down to a few centimetres object in LEO [4]. Furthermore, a bistatic radar system known as GRAVES [5], based in France is used to monitoring space debris and the German Tracking and Imaging Radar (TIRA) system can estimate orbital parameters target shape and size of space debris using signal processing techniques [6]. US Space Fence is a space surveillance system designed by and developed by Lockeed Martin for US Air Force to track down artificial space objects and debris orbiting the Earth [7]. This system, will allow the detection of much smaller microsatellites and debris, than current systems and also improve the timeliness which space events can be detected avoiding any threads for existing satellites. Space fence operation is expected to operate in 2018.

This paper proposes a new potential system for space object detection and characterisation with a passive space-borne bistatic radar installed on one or more cubeSATs flying in low Earth orbit. Exploiting any RF source signals emitted from higher altitude illuminators, this system could detect space debris orbiting the Earth, at a lower cost and comparable performance as existing systems. In contrast with ground-based systems, the proposed system overcomes the limitations of signal attenuation due to long distances, atmosphere and bad weather, without increasing mission and system complexity. Due to shorter distances, a good Signal to Noise Ratio could be achieved with simpler hardware and cost and also integration time could be significantly increased due to lower relative velocities involved. The minimum detectable target size, is the key performance metric for this proposed system.

The remainder of the paper is organized as follows. Section II describes the kinematic model and the proposed radar system configuration. In Section III, the integration gain factor is introduced and analysed. Section IV presents simulation results depending on the main parameters that affect the performance of the system such as altitude of the illuminator, carrier frequency etc. Section V concludes the paper.

\section{Kinematic Model}

The physical structure of the system is shown in Figure 1. Flying at low altitude, the cubeSAT carrying the radar 
receiver unit, is below space debris exploiting higheraltitude illuminators $\left(\mathrm{T}_{\mathrm{x}}\right)$. Also, this structure makes forward scattering feasible. Forward Scattering (FS), is a phenomenon occurred in a special bistatic radar configuration when the target is crossing the transmitterreceiver baseline (L) [8]. At that point, the target's radar cross section (RCS)* observed by the receiver is enhanced and depends only on the physical shape and silhouette of the target [9]. Therefore, the detection performance of the system could be significantly increased exploiting FS.

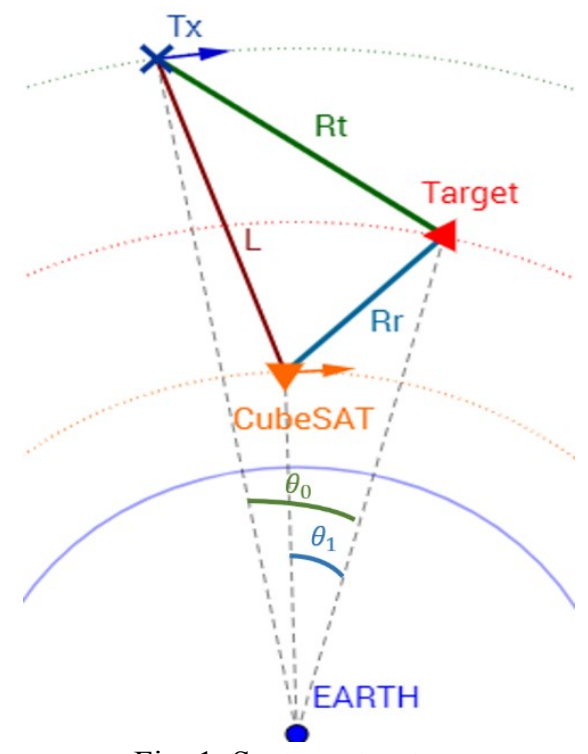

Fig. 1. System structure

System performance could be evaluated depending on the minimum detectable object or minimum detectable radar cross section. Therefore, using the radar range equation (RRE) (1) for bistatic radar [10], detection performance can be investigated and support the feasibility of the proposed system.

$$
\sigma_{b}=\frac{(4 \pi)^{3} R_{t}^{2} R_{r}^{2} k T_{0} B_{r} F}{P_{t} G_{t} G_{r} \lambda^{2} L_{s}} \frac{S N R}{\sqrt{N} G_{s p} G_{L N A}}
$$

where $\mathrm{R}_{\mathrm{t}}$ and $\mathrm{R}_{\mathrm{r}}$ are Transmitter-to-Target and Target-toReceiver distance respectively and $N$ is the number of pulses integrated depending on the integration time. $\mathrm{G}_{\mathrm{LNA}}$ and $\mathrm{G}_{S P}$, are gains introduced by low noise amplifier and signal processing i.e.: matched filter, $\mathrm{P}_{\mathrm{t}}$ is the transmit power, $\mathrm{G}_{\mathrm{t}}$ and $\mathrm{G}_{\mathrm{r}}$ are the transmitter and receiver antenna gains, $\lambda$ is the signal carrier wavelength, $\sigma_{\mathrm{b}}$ is the bistatic radar cross section (measured in $\mathrm{m}^{2}$ ), $k$ is the Boltzmann's constant, $B$ is the receiver effective bandwidth, $L_{s}$ are other system loses, $F$ is the receiver effective noise figure and $T_{0}$ is the noise reference temperature (generally equal to $290 K)$. The value of the noise reference temperature presupposes that a thermal control is applied to the system in order to stabilize the temperature at the receiver due to the fact that system operates in thermosphere.

For this analysis, a two dimension kinematic model for circular orbits is used for simplicity reasons. The main parameters needed for a circular orbit determination are shown in Figure 2. Assuming objects orbiting the Earth in perfect circular manner, orbital parameters can be extracted and used for the kinematic model of the system.

In a completely circular orbit, object's altitude determines its angular velocity orbiting the Earth as:

where,

$$
\omega_{o b j}=\sqrt{G \frac{M_{e a r t h}}{R_{o b j}^{3}}}
$$

$$
\boldsymbol{R}_{\text {obj }}=\boldsymbol{R}_{\text {earth }}+\boldsymbol{O b j _ { A l t }}
$$

To evaluate the proposed system achievable performance, the worst case scenario is be considered which is when the target is orbiting the Earth in an opposite direction with cubeSAT and their orbits are coplanar (Figure 1). This scenario guarantees the minimum observation time between illuminator, target and the cubeSAT.

By fixing the three object's altitude, their orbit behaviour is known. Transmitter-to-Target and Target-to-Receiver distances can easily be evaluated, using equations (3), (4) and (2).

$$
\begin{aligned}
& R_{t}=\sqrt{R_{T x}^{2}+R_{T r g}^{2}-2 R_{T x} R_{T r g} \cos \left(\left[\omega_{T r g}-\omega_{T x}\right] t+\theta_{0}\right)} \\
& R_{r}=\sqrt{R_{R x}^{2}+R_{T r g}^{2}-2 R_{R x} R_{T r g} \cos \left(\left[\omega_{R x}-\omega_{T r g}\right] t+\theta_{1}\right)}
\end{aligned}
$$

where $\theta_{0}$ and $\theta_{1}$ are the initial angle phases of the illuminator-target and target-cubeSAT respectively.

From equation (3) and (4), it is shown that Transmitterto-Target and Target-to-Receiver distances can be really affected due to relative angular velocities. In this case, these variables are changing rapidly due to the opposite orbit direction of the objects and thus, coherent integration time cannot be assumed. Coherent and noncoherent integration will be discussed in the next section.

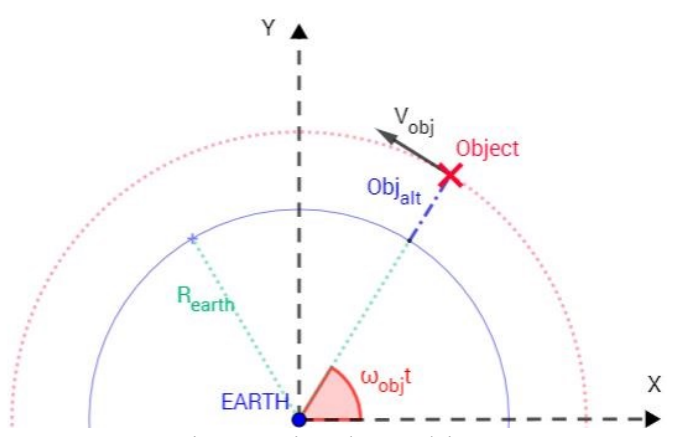

Fig. 2. Circular Orbit 


\section{Integration Time}

In this section, the term Integration Time for radar processing will be discussed. Detecting targets in a real world with noise, a good SNR at the receiver of the radar must be achieved in order to minimize miss-detection. Averaging returns received by the same target will linearly increase the SNR at the receiver, allowing detection to be as reliable as could be. When both the amplitude and phase of the returns are used in the processing so all received pulsed ended up added in phase, then coherent integration assumed. Coherent integration of $N$ pulses could result to an increase of SNR by $N$ times. Non coherent integration, does not averaging target returns always in phase and thus the gain is always less than coherent integration. Non coherent integration of $N$ pulses provides an integration gain around $N^{0.5}$ [11].

Integration gain is important for system's detection performance. Taking the worst case scenario mentioned above, the minimum observation time must be evaluated. Assuming the surveillance antenna beamwidth facing upwards, as illustrated in Figure 3, the time needed for the target to cross the arc length of the along-track beamwidth may be considered as the maximum time a cubeSAT can incoherently integrate pulses.

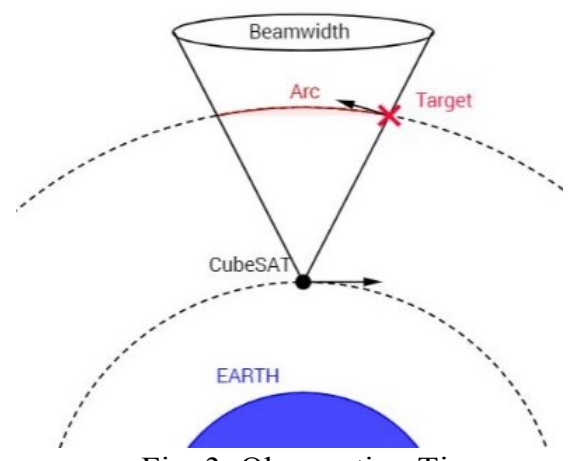

Fig. 3. Observation Time

This parameter (the arc length) depends only on the altitude difference between cubeSAT and target.

Receiver's antenna beamwidth can be calculate as:

$$
\mathbf{\Omega}=\frac{\lambda}{\mathrm{D}} \boldsymbol{r a d s}
$$

Where $D$ is the receiver along track antenna size.

This dependence is shown in Figure 4 where cubeSAT's altitude is fixed at $400 \mathrm{~km}$, receiver antenna beamwidth at $0.044^{\circ}$ with antenna length $(D)$ of $0.5 \mathrm{~m}$.

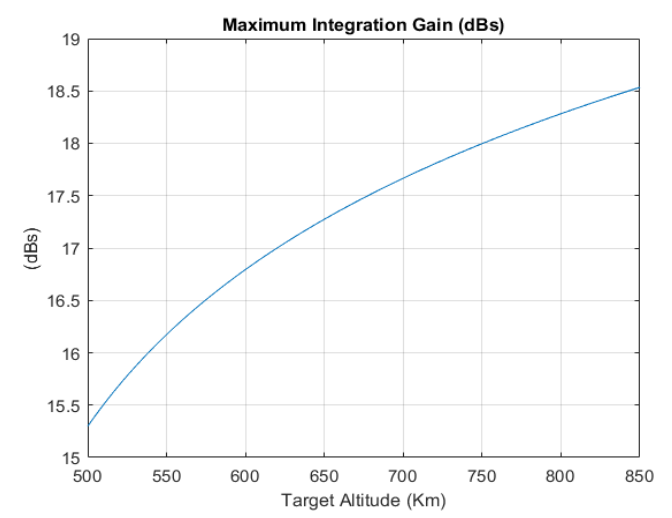

Fig. 4. Maximum Integration gain VS Target altitude

As expected, integration gain is proportional to the altitude difference between the cubeSAT and the target. For a fixed-altitude cubeSAT at low earth orbit $(400 \mathrm{~km})$, an integration gain of at least $18 \mathrm{~dB}$ can be achieved for a target's altitude higher than $750 \mathrm{~km}$.

In Figure 5, the spatial distribution of space objects is illustrated, according to 2011 NASA report to the United Nations office. As it can be seen, a peak density is located at around $800 \mathrm{~km}$ of altitude. Therefore, for this analysis, $1 s$ of integration time can be assumed feasible.

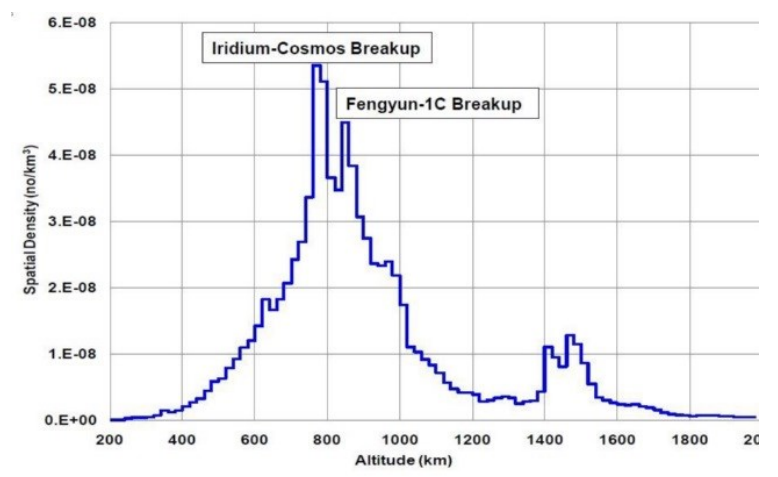

Fig. 5. Spatial Density of Space Object, source NASA 2011 [12].

\section{Simulation and Results}

In this section some simulation results will be reported. System performance will be discussed using different illuminators, with different carrier frequencies and altitudes. Three different satellites are used for this analysis. The first is the Haiyang-2A (HY2A), which is a second generation satellite series for ocean monitoring approved by the China National Space Administration (CNSA) in Beijing [13]. The second is Jason-3, which is an altimetry mission [14], the third illuminator considered is a generic Global Star satellite dedicated to satellite phone and low-speed data communication [15]. All these 3 satellites are chosen in order to provide an analysis with a wide range of transmission frequencies 
and altitudes. Table 1 illustrates all three satellites parameters.

Table 1. Link Budget Parameters

\begin{tabular}{|c|l|l|l|l|}
\hline & Descr. & HY2A & Jason 3 & G. Star \\
\hline $\boldsymbol{\lambda}$ & Wavelength & $22 \mathrm{~mm}$ & $56.6 \mathrm{~mm}$ & $120 \mathrm{~mm}$ \\
\hline $\mathbf{G r}$ & Receiver gain & $34 \mathrm{Db}$ & $42 \mathrm{~dB}$ & $19.30 \mathrm{~dB}$ \\
\hline $\mathbf{E I R P}$ & EIRP & $52.5 \mathrm{~dB}$ & $52 \mathrm{~dB}$ & $37 \mathrm{~dB}$ \\
\hline $\mathbf{B}$ & Bandwidth & $320 \mathrm{MHz}$ & $\begin{array}{l}320 \\
\mathrm{MHz}\end{array}$ & $\begin{array}{l}16.5 \\
\mathrm{MHz}\end{array}$ \\
\hline $\mathbf{G s p}$ & Sig. Pr. gain & $45.15 \mathrm{~dB}$ & $\begin{array}{l}44.11 \\
\mathrm{~dB}\end{array}$ & $52.04 \mathrm{Db}$ \\
\hline GLNA & LNA gain & $40 \mathrm{~dB}$ & $42 \mathrm{~dB}$ & $50 \mathrm{~dB}$ \\
\hline $\mathbf{F}$ & $\begin{array}{l}\text { Radar noise } \\
\text { fig. }\end{array}$ & $10 \mathrm{~dB}$ & $10.5 \mathrm{~dB}$ & $12.5 \mathrm{Db}$ \\
\hline Alt & Altitude & $1000 \mathrm{~km}$ & $1336 \mathrm{~km}$ & $1400 \mathrm{~km}$ \\
\hline
\end{tabular}

\begin{tabular}{|c|c|c|}
\hline $\boldsymbol{k}$ & Boltzmann Const. & $1.38 \times 10^{-23} \mathrm{~J} / \mathrm{K}$ \\
\hline $\mathbf{T}_{\mathbf{0}}$ & Noise reference Temp. & $290 \mathrm{~K}$ \\
\hline $\mathbf{L}_{\mathbf{s}}$ & Loss Factor & $0 \mathrm{~dB}$ \\
\hline SNR & SNR at the receiver & $10 \mathrm{~dB}$ \\
\hline
\end{tabular}

Using equations (3) and (4), the parameters in table 1 and a time step of $0.1 \mathrm{~s}$, the minimum detectable radar cross section is evaluated using RRE (1). For every time step the distances $R_{t}$ and $R_{r}$ were calculated assuming one second of integration time. CubeSAT altitude is fixed at $400 \mathrm{~km}$ and target's altitude at $750 \mathrm{~km}, 850 \mathrm{~km}$ and $950 \mathrm{~km}$. Figures 6-8, show the results obtained in terms of minimum detectable RCS for the three satellites at the three altitudes.

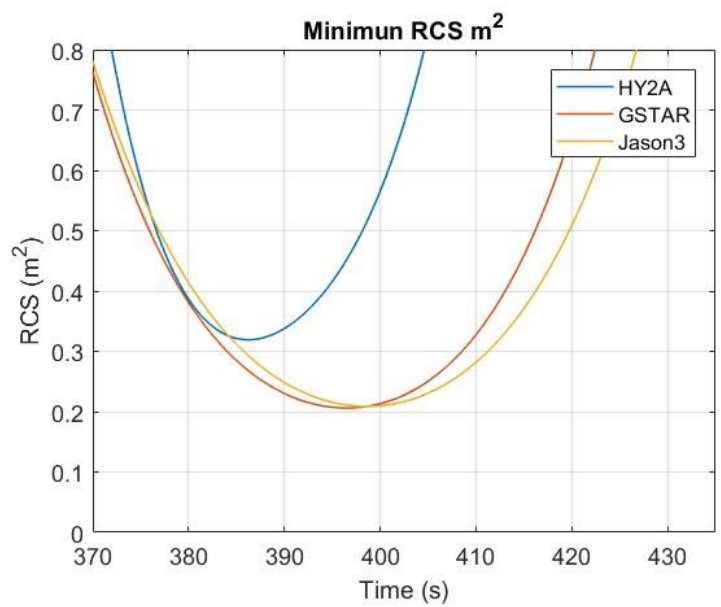

Fig. 6. Minimum Detectable RCS: Target@750km

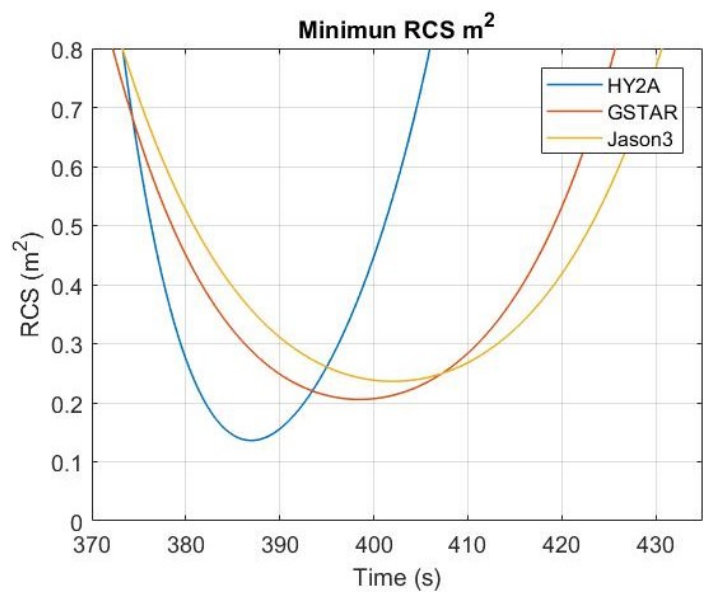

Fig. 7. Minimum Detectable RCS: Target @ 850km

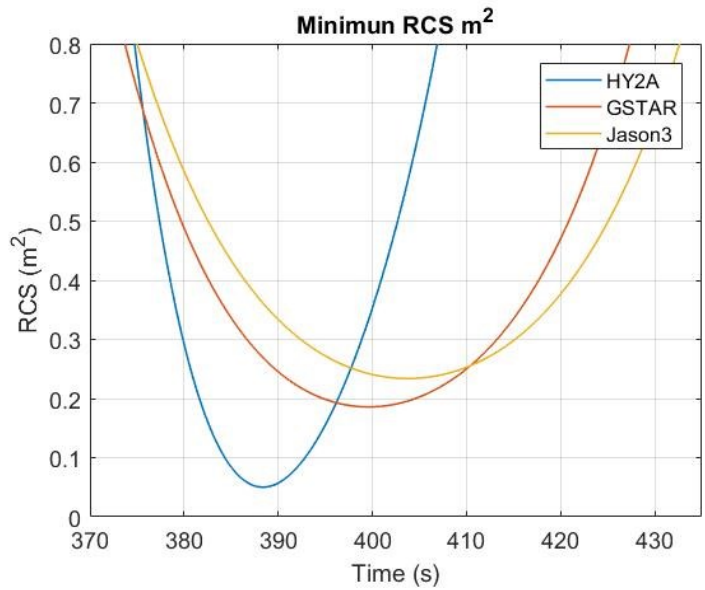

Fig. 8. Minimum Detectable RCS: Target @ 950km

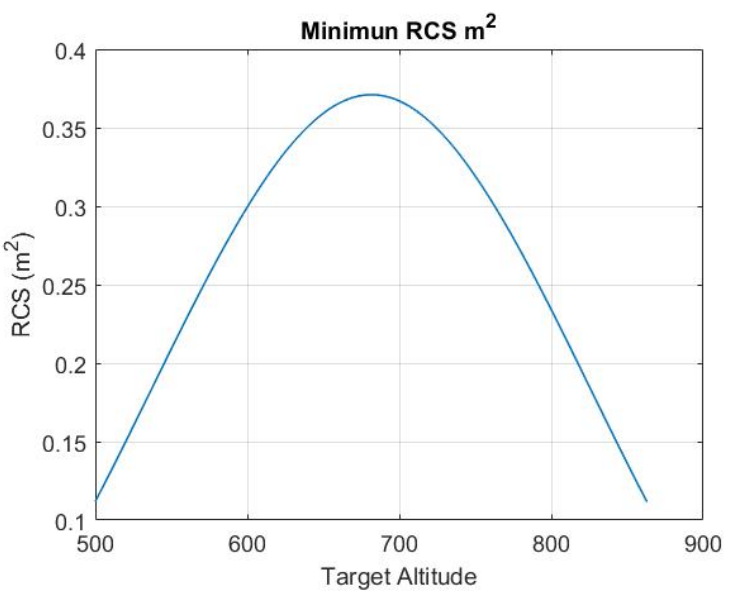

Fig. 9. Minimum Detectable RCS: Target 500km$900 \mathrm{~km}$

As it can be seen from Figures 6-8, the detection performance of the system are promising. For all three different illuminators, a minimum RCS of less than $0.4 \mathrm{~m}^{2}$ achieved. Furthermore, is worth mentioning the drop of 
minimum RCS noticed, as the target's altitude increases. Figure 9 shows this relationship, illustrating the minimum detectable RCS for the HY2A illuminator, with target's altitude varying from $500 \mathrm{~km}$ to $900 \mathrm{~km}$. Illuminator and cubeSAT are orbiting at $1000 \mathrm{~km}$ and $400 \mathrm{~km}$ respectively in this latter simulation.

Clearly, optimum detection performance is achieved when target's altitude is closer to the one of two system components. This also can be observed from RRE (1), where the distance product $\left(R_{t} R_{r}\right)^{2}$ is maximum when the two distances are equal. Therefore, cubeSATs altitudes could be chosen properly in order to achieve optimum system performance.

\section{Conclusion and Future work}

In this paper a feasibility study for a new space-borne radar system is presented. A simple and low cost solution for space debris detection in low Earth orbit is proposed where one or more cubeSATs equipped with a small antenna could exploit any RF source from higher altitude satellites to detect space objects. Simulation results showed that system performance could be promising, detecting space objects down to few centimetres in LEO. Moreover, the relatively low distances from the targets and the absence of signals attenuation due to atmospheric and other factors, significantly contributed to these results. An approach to estimate the maximum integration gain is presented, showing that even with high orbital velocities, this system manages to reduce relative velocities and increasing the time where target receiver and illuminator could observe each other.

The potential of this system could be further improved and investigated. In terms of detection, integration gain could be enhanced using a rotating antenna which could dynamically rotate respect to target's orbits. Furthermore, exploiting forward scattering, target classification may be possible using Shadows and Doppler signatures. Finally, using a cubeSAT network, target localization could be achieved extracting orbital parameters needed space object catalogue.

\section{Footnotes}

* Radar Cross Section is a measure which provides information about the target reflectivity in the direction of radar's receiver and is measured in units of area (e.g. $\mathrm{m}^{2}$ ). Target's material, shape and the angle of incident wave affects the value of the RCS and does not exclusively depend on the size of target [16].

\section{References}

[1]"About space debris", European Space Agency, 2017. [Online].Available:http://www.esa.int/Our_Activities/Operatio ns/Space_Debris/About_space_debris (accessed 15.07.17).
[2]2017.[Online].Available:https://ntrs.nasa.gov/archive/nasa/ casi.ntrs.nasa.gov/20100002023.pdf .

[3]N. Smirnov and E. Institute, Space debris: Hazard evaluation and mitigation. London: Taylor \& Francis, 2002.

[4]"Space Situational Awareness", European Space Agency,2017.[Online].

Available:http://www.esa.int/Our_Activities/Operations /Space_Situational_Awareness. (accessed 25.08.17).

[5]J. Ender, L. Leushacke, A. Brenner and H. Wilden, "Radar techniques for space situational awareness", International Radar Symposium (IRS), pp. 21-26, 2011

[6]"Space observation radar TIRA - Fraunhofer FHR", Fraunhofer Institute for High Frequency Physics and Radar Techniques FHR, 2017. [Online].Available:https://www.fhr.fraunhofer.de/en/the -institute/technical-equipment/Space-observation-radarTIRA.html. (accessed 15.07.17).

[7]"Space Fence- Lockheed Martin", Lockheedmartin.com, 2017.[Online].Available: http://www.lockheedmartin.com/us/products/spacefence.html. (accessed 15.07.17).

[8]J. Scheer and W. Melvin, Principles of modern radar, 3rd ed. Scitech, 2014.

[9]N. Willis, Bistatic radar. Norwich, NY: Knovel, 2006, pp. 151-153.

[10]N. Willis, Bistatic radar. Norwich, NY: Knovel, 2006.

[11]M. Richards, J. Scheer and W. Holm, Principles of Modern Radar. SciTech Publishing, 2010, pp. 536-538.

[12]"Space debris | Wikiwand", NASA, 2011. [Online]. Available: http://www.wikiwand.com/en/Space_debris.

[13]"HY-2A-eoPortal Directory - Satellite Missions", Directory.eoportal.org, 2017. [Online]. Available:https://directory.eoportal.org/web/eoportal/sat ellite-missions/h/hy-2a. (accessed 28.07.17).

[14]"Jason-3 - Satellite Missions - eoPortal Directory", Directory.eoportal.org, 2017. [Online]. Available:https://directory.eoportal.org/web/eoportal/sat ellite-missions/j/jason-3. (accessed 28.07.17).

[15]2017.[Online].Available:https://www.ic.gc.ca/eic/site/smtgst.nsf/vwapj/smse00205-globalstarannexa.pdf/\$FILE/smse00205-globalstar-annexa.pdf.

[16]M. Richards, J. Scheer and W. Holm, Principles of Modern Radar. SciTech Publishing, pp. 219-230 